\title{
Arbor
}

\section{Blas Cabrera, las Academias y su Tiempo}

\section{Ángel Martín Municio}

Arbor CLXXIX, 706 (Octubre 2004), 579-593 pp.

Ya se toca la fecha del cincuentenario de la desaparición de Don Blas Cabrera (1878-1945). Su vida se acompasó con la misma historia de la ciencia española en unas cuantas décadas del comienzo de este siglo. Y para hacer de la tradición memoria venimos a la tierra que le vio nacer a pregonar lo que él supuso en la marcha cansina de nuestra ciencia; a evocar lo que él significó como punto de inflexión de la Física española; a exaltar lo que Cabrera representó en el avance de la ciencia hacia su confluencia con el humanismo y la cultura; a ejemplificar en él una de las actitudes más completas y múltiples de las que un hombre de ciencia puede mostrar, e incluso adelantándose un tanto a ese pregón más actual de la presencia de la ciencia en la sociedad.

Para evitar, pues, que el recuerdo se difumine en la simple melancolía o, peor aún, se pierda en el silencio cómplice, que dé la razón al historiador cuando, al señalar la relación de los españoles con su historia, dice que sus hazañas han caído por falta de plumas para alabarlas. Para enriquecer la historia con nuestro recuerdo, aglutinados por el profesor González de Posada, hemos conmemorado este cincuentenario, aquí y allá, la Academia de la que formó parte, los discípulos, los amigos, las gentes de su tierra.

Se dice muchas veces que la verdad es hija del tiempo, y hasta Cervantes asegura que los tiempos mudan las cosas. Pero, ni las cosas, ni mucho menos las verdades, pueden mudar con el tiempo. Lo que sí ocurre es que la distancia hace menos imperfecta y más global la contemplación de los objetos. Desde las nuevas coordenadas se aprecia mejor lo que de permanente y de continuo hay en la naturaleza de los hombres. 


\section{Ángel Martín Municio}

Continuidad que une el saber de las generaciones pasadas con el de la presente; y esa es la función perfeccionadora del tiempo al conservar pertinazmente los recuerdos. Yo, que, por razón de ese tiempo, no pude conocer a Don Blas Cabrera he tenido que acudir a estrujar las fuentes originales de la historia para poder concluir, tan objetiva como inequívocamente, que él supuso la punta de lanza de ese segundo intento de incorporación de nuestra ciencia a la ciencia europea en el ecuador de la primera mitad de nuestro siglo. Yo que, a medio siglo de distancia, he ocupado la presidencia de la misma Academia de Ciencias que él dirigió, quiero izar, además, en este acto un recuerdo afectivo que se fusione con ese intenso sentimiento añorante de sus últimos años. ¡Que no falte la gratitud con que las nuevas generaciones aceptamos aquel ofrecimiento de Cabrera, el 4 de marzo de 1923, ante S.M. el Rey D.Alfonso XIII: «Espero que al final de vuestra vida, que será también el de mi generación, la España científica que hoy apenas encontráis en embrión, haya llegado al lugar que tiene el inexcusable deber de ocuparn! ¿Qué en la evocación del recuerdo científico de Cabrera tampoco falte la emoción de su ejemplaridad personal y del sacrificio de los últimos años de su vida!

Acabo de calificar la actividad de Cabrera como de punto de inflexión en la historia de la ciencia española, en su segunda aproximación a la ciencia europea. En efecto, las gentes de la Ilustración llevaron a cabo la primera convergencia española hacia la ciencia mundial del XVIII; la primera presencia española en lo que hoy llamaríamos política científica. Ya en el ambiente ilustrado de la época existieron planteamientos tales como los de la participación política del gobierno en el desarrollo de la ciencia, las relaciones internacionales, el fomento de la industria, la transferencia de tecnología, la conexión investigación-industria, la participación de la iniciativa privada, la contratación de personal extranjero, las repercusiones económicas de la investigación, la atención al equipamiento instrumental, los temas prioritarios, etc. Situaciones todas ellas que, con los mismos nombres o bajo etiquetas más rimbombantes, como la infraestructura, los sistemas de I+D, etc., se siguen incorporando dos siglos después a nuestra sociología de la ciencia. Lo más importante es que bajo este ambiente fue cuando los españoles realizaron una de sus mejores aportaciones a la historia de la química y de la metalurgia: en 1783, los riojanos hermanos Juan José y Fausto Elhúyar descubrieron el elemento 75 , el wolframio, en muestras de las minas de Sajonia; el madrileño Andrés Manuel del Río, graduado por Alcalá de Henares, descubrió, en 1801, el eritronio, elemento 23 , más tarde rebautizado como vanadio; y, anteriormente, en 1736, Antonio de Ulloa, como miembro de la expedición 
hispanofrancesa dirigida por La Condamine, descubrió el platino. Para situar el interés de estos acontecimientos convendrá situarlos en el contexto de los hechos de la ciencia europea. Así, los hermanos Elhúyar habían previamente estudiado en París, en la época de los descubrimientos de Lavoisier y Priestley sobre el oxigeno y la combustión; mientras ambos viajaban por los centros de investigación de Europa, Coulomb descubrió la electricidad, Scheele aisló el ácido cianhídrico, y Cavendish llevó a cabo la síntesis del agua; y mientras iniciaban su larga estancia americana tenía lugar la reforma de la nomenclatura de la química a manos de Lavoisier, Guyton de Morveau, Fourcroy y Berthollet.

Primer esfuerzo serio de la ciencia española, casi exclusivamente de la química, y primeros resultados hacia la convergencia con la ciencia europea, en coincidencia con un periodo de estabilidad política, de creación de instituciones científicas, y de protección a las manifestaciones culturales. Desarrollo científico que había enseguida de anularse al desaparecer las imprescindibles condiciones políticas y económicas que lo sustentaran. Por ello, y para justificar esta posterior decadencia, veamos que sucedió en la ciencia europea y en los avatares nacionales durante el siglo XIX.

En la primera década del siglo XIX quedaron establecidas las primeras leyes de la química, la ley de Proust de las proporciones definidas, la ley de Dalton de las proporciones múltiples e hipótesis atómicas y la ley de Gay-Lussac sobre los volúmenes de los gases; Davy aisla el sodio, el potasio y el cloro; Volta desarrolla la primera pila eléctrica; Göthe publica la Teoría de los colores. España sufrió la derrota de Trafalgar en 1805, el Motín de Aranjuez en 1808, la Guerra de la Independencia durante el cuatrienio 1808-12, y las Cortes de Cádiz en 1810.

En la segunda década, Berzelius aisló el silicio (1811) y Avogadro estableció la ley sobre las densidadés de los gases; Laplace publicó su obra Teoría analítica de probabilidades (1812) y Cauchy el Estudio sobre integrales definidas (1814); Fresnel llevó a cabo sus primeros trabajos sobre la luz (1813) y la difracción (1816); Magendie realizó sus experimentos sobre fisiología animal. En 1818, Maria Shelley publicó la obra que dio comienzo a la literatura de ciencia ficción: Frankestein. España, en 1811, reconoció la independencia de Venezuela, y, en 1816, la de la Argentina.

En la década de los 20, Ampère estableció la teoría matemática de la electrodinámica (1826); Fourier publicó la Teoría analítica del calor y Fresnel lo hizo de la Teoría ondulatoria de la luz; Wöhler llevó a cabo la 


\section{Ángel Martín Municio}

primera síntesis de una sustancia orgánica: la urea. En España, mientras tanto, transcurrió el trienio liberal (1820-1823), el absolutismo, y se ofrecía la perspectiva de las recurrentes Guerras Carlistas. El mismo año del compromiso de Vergara, 1839, Liebig diseñó la teoría de la fermentación y Boole la de la transformación analítica. De aquí a mediados del siglo, Joule (1842) estableció el principio de conservación de la energía y Wheatstone (1843) inventó el puente de su nombre; Bessel (1840) midió por primera vez la distancia de una estrella, y Faraday (1846) intuyó la naturaleza electromagnética de la luz. En estos mismos años, Comte publicó el Discurso sobre el espíritu positivo, y Marx y Engels lo hicieron del Manifiesto del partido comunista; a la vez que, entre Washington y Baltimore se establecía la primera línea de telégrafos (1844) y se llevó a cabo la primera anestesia total (1846). En esta época se producen en España ligeros atisbos de una cierta estructuración en los campos de la instrucción pública, con el Plan Pidal de 1845 y, una docena de años después, la Ley Moyano que gobernó la educación de los españoles hasta bien entrado el presente siglo. En el año 1847 se refundó, en su versión actual, la Real Academia de Ciencias que heredaba, ciertamente con altibajos y cambios de nombre, la tradición fundacional de la Academia de Matemáticas de Madrid, creada por Felipe II a finales del siglo XVI (1582) y que tuvo por primer presidente al arquitecto Herrera.

Toda una colección de eminentes personajes de la ciencia jalona el tercer cuarto del siglo XIX. Entre otros, Bunsen, descubridor del magnesio (1852); Riemann, instaurador del cálculo integral; Bernard (1855) y Pasteur (1857), fundadores de la fisiología de los seres vivos; Kekulé (1858), creador de la teoría de la valencia química, y Berthelot (1860), autor de la primera síntesis del acetileno; Solvay (1863), diseñador del procedimiento que lleva su nombre de fabricación de la sosa; Maxwell (1864), artífice de la teoría dinámica de los campos electromagnéticos; Mendel (1865), investigador de la hibridación de las plantas; Nobel (1866), descubridor de la dinamita; Mendeleiv (1869), organizador de los elementos químicos en el cuadro periódico de acuerdo con sus propiedades; Le Bel y Van't Hoff, forjadores de la estereoquímica y la química molecular; Boltzmann (1877), autor de la teoría cinética de los gases. Cuarto de siglo que, al lado de la publicación de El origen de las especies (1859) de Darwin, y de la Introducción al estudio de la medicina experimental de Claude Bernard, vió la aparición de Madame Bovary (1857) de Flaubert, de Humillados y Ofendidos (1861) de Dostoievski, de Los Miserables (1862) de Victor Hugo, de Guerra y Paz (1869) de Tolstoi, de Una temporada en el Infierno (1873) de Rimbaud. La historia se planta así, con es- 


\section{Blas Cabrera, las Academias y su Tiempo}

tos antecedentes, en 1878, fecha en que, en Lanzarote, nació Don Blas Cabrera. Pero, entre nosotros volvíamos a las andadas con los pronunciamientos de 1854; la revolución burguesa de 1868, la Constitución del 69 y la regencia del general Serrano (1868-1871); el reinado de don Amadeo (1871-1873), la Primera República (1873-1874), el pronunciamiento de Martínez Campos y la Restauración.

A propósito de aquellas "andadas" quiero traer a colación unos párrafos pronunciados el 17 de abril de 1910 por Don José Echegaray, con motivo de la contestación académica en el ingreso de Don Blas Cabrera en la Real Academia de Ciencias. Decía de esta manera Echegaray, al glosar los momentos más hondamente grabados de la vida intelectual del nuevo académico: "Recuerdo a mi vez el momento, para mí solemne, ya bien lejano, en que esta Academia me abrió sus puertas, y recuerdo el discurso que en aquella ocasión leí ante vosotros: ante vosotros no, ante otros compañeros que ya pasaron. Y como veréis, este recuerdo es oportuno. Eran tiempos aquellos de inmensa agitación en España, porque acercábase el mes de septiembre de 1868 y se sentían los estremecimientos de la gran convulsión revolucionaria. Eran tiempos de lucha y de combate, y mi discurso, aunque habia de ser puramente científico, de aquellos combates $y$ de aquellas luchas hubo de resentirse: cuando tiembla el suelo, tiemblan los palacios y tiemblan las chozas. Afirmaba yo que, desde los árabes hasta el día, España, tan grande y tan fecunda en otras esferas, había sido estéril en lo que se refiere al orden de las matemáticas puras, sin que, para vergüenza y desesperación nuestra, pudiéramos contar en nuestra gloriosísima historia con un solo matemático de primer orden, como si el cerebro de nuestra raza fuera impotente y obtuso para las altas lucubraciones de la ciencia de Newton, Descartes y Leibnitz. Naturalmente, yo atribuía esta desdicha a causas políticas, al absolutismo y a la intolerancia, y de este modo tomaba mi peroración notas batalladoras de entre aquéllas que ya vibraban en el aire. Naturalmente, repito, mi rotunda y cruel afirmación dio motivo a muchas protestas y a muchos artículos en los periódicos de críticos, que demostraban de este modo un patriotismo, siempre respetable y simpático; pero casi todos mostraban a la vez una ignorancia inexcusable y triste en la materia de que se trataban. Tomaba parte, ciertamente, Echegaray en la tan famosa como inútil polémica sobre la ciencia española. También Cabrera, como más adelante comentaremos, habría de tomar parte en ella, con argumentos semejantes a los de Echegaray.

Bajo estas condiciones innegables, difícil habría de ser la penetración en España de las corrientes europeas del pensamiento, tan sólo favoreci- 


\section{Ángel Martín Municio}

da por un incipiente triunfo de la libertad de expresión que, aún con gran retraso, lograba incorporar las realizaciones más características del pensamiento europeo decimonónico. Así, por ejemplo, solamente se dieron en España noticias indirectas de los grandes filósofos del idealismo germánico, Fichte, Schelling y Hegel. La primera versión del pensamiento de Fichte, los Principios fundamentales de la teoría de la ciencia, vio la luz en Zaragoza un siglo después de su inicial publicación; la Filosofía del derecho se conoció en España con un retraso de más de medio siglo sobre la fecha de su aparición; Schelling permaneció prácticamente ignorado. Y, en un intento de superar estos males, bajo la regencia de Espartero se encargó a Sanz del Río de contactar con los krausistas y extender luego en España sus doctrinas, plenas de austeridad y rigor ético y regidas por el predominio de la autonomía moral del individuo. Bajo estas normas había de nacer en 1876, la Institución libre de enseñanza, con el objeto de crear las condiciones necesarias para la realización del ideal krausista. Institución que tanto había de influir sobre nuestro ulterior desarrollo cultural y científico. A instancias, precisamente de la Institución libre de enseñanza, creó el Gobierno, en 1907, la Junta para Ampliación de Estudios e Investigaciones Científicas con objeto de promover la investigación científica nacional. A los pocos años, en 1910, la Junta contó con el primer laboratorio de investigación física. Poco tiempo antes, en 1903, se había fundado la Sociedad Española de Física y Química, bajo la presidencia de Echegaray. Junta de Ampliación de Estudios y Sociedad de Física y Química que entroncan ya, en la primera década del siglo XX, con la entrada de Cabrera en la actividad científica de la física. Cuatro hechos enmarcan esta inicial presencia de Cabrera en la física española: El doctorado en Ciencias Físicas, en 1901, por la Universidad de Madrid; la participación, en 1903, como socio fundador, en la creación de la Sociedad Española de Física y Química; enseguida, en 1905, es Catedrático de Electricidad en la Universidad de Madrid; y, en 1910, el ingreso, ya aludido, en la Real Academia de Ciencias. Son los años en que tiene lugar la maduración de la física moderna. En los años finales del XIX, Röntgen descubrió los rayos X (1895) y Becquerel lo hizo de la radiactividad (1896); Zeemann describió el efecto del campo magnético sobre la luz (1896); los Curie, en 1898, descubrieron el polonio y el radio, y, en el último año del siglo, Rutherford lo hizo de los rayos alfa y beta. Y, entrando ya el presente siglo, Planck estableció la teoría de los "cuantos»; Einstein, en 1905, publicó su famoso artículo "Relatividad restringida, relación masa-energían; Geiger concibió un detector de partículas; y Bohr y Rutherford propusieron un modelo para la estructura del átomo. 


\section{Blas Cabrera, las Academias y su Tiempo}

Es importante subrayar el enorme contraste entre la brillante situación de la ciencia europea, y de la física en particular, cuando comienza el siglo XX, y la extraordinariamente pobre presencia española en todos los campos, más aún, quizá, en el de la física. Contraste que tiene que servir para valorar en su justa medida el gran y múltiple esfuerzo de Blas Cabrera para iniciar la incorporación de España a la creación científica de la física. A este contraste y a las pobres condiciones de la investigación física española se referiría Cabrera en muchas ocasiones. En una de ellas, con motivo de su ingreso en la Academia Española, en enero de 1936, puntualizaba: "Es necesario llegar a los primeros años del siglo actual para que se reemprendiera el camino iniciado por los coetáneos de Carlos III, precisamente gracias a la obra que comenzaron los hombres beneméritos que presidió Cajal. Pertenezco yo -continuaba Cabrera-a la última generación que se encontró huérfana de una tradición que le orientara por camino real en los años más difíciles para las grandes resoluciones que pueden guiar toda una vida y por ello mismo expuesta a la pérdida de las ventajas que por compensación se ofrecen a la actividad por la frescura imaginativa que sugiere métodos ricos en frutos para la propia labor. Por mi suerte, tuve en cambio las ventajas derivadas de la acción directa de Ramón y Cajal».

Nada fácil, a todas luces, liderar este reemprender el camino; esta inicial incorporación que obligaba a estar presente en todo ese conjunto de variadas acciones simultáneas exigibles, entonces y ahora, para llevar a cabo la coordinación científica y administrativa, nacional e internacional, de la investigación. Cabrera tuvo que iniciar esta incorporación desde los más variados ángulos; desde la elección del tema objeto de su propia observación experimental, a la creación del equipo de trabajo y de la escuela investigadora con sus múltiples actividades de formación y de gestión; desde la estructuración temática y humana de nuevos centros de investigación física objeto de su dirección personal al ejercicio del gobierno administrativo de la investigación, a la confección de las publicaciones nacionales e internacionales y a las conexiones con los centros extranjeros; desde el ejercicio director de instituciones del más elevado prestigio nacional -Universidades, Academias, Sociedades científicas- a su presencia en destacados organismos y comisiones internacionales; desde las publicaciones científicas en revistas especializadas al ejercicio trascendental de la divulgación y la educación científicas. Si en la actualidad, con las indudables facilidades de todo tipo en el momento presente y con las ideas en marcha, son muy escasas las gentes de la ciencia capaces de abarcar con rigor todo este conjunto de actividades, tendremos que ren- 


\section{Ángel Martín Municio}

dirnos a la inteligencia, a la capacidad y al entusiasmo de nuestros predecesores, tal es el caso singular de Blas Cabrera, impulsores de ese segundo intento de convergencia científica europea.

Fue, pues, Cabrera protagonista principal de la transformación operada en la física española en las primeras décadas del siglo XX. No se le puede pedir más al hombre que intentó, quizá como ningún otro de su generación, que España llegase al lugar que tenía el inexcusable deber de ocupar. Fue, en su época, uno de los científicos que dieron el paso gigante del hablar de al trabajar sobre. Al margen de cualquier biografía pormenorizada, yo voy a situar fervorosamente a Cabrera en el espacio y en el tiempo con motivo de sus intervenciones académicas en la Española y en la Real de Ciencias, reflejo de la tenacidad con que se enfrentó a la naciente modernidad de nuestra ciencia. Lo voy a hacer amparándome en unas bellas imágenes de Emilio Lledó sobre el lenguaje: "La palabra es, para la existencia, el alimento de su maduración y el inagotable caudal de su libertad para pensar. No hay otra posibilidad de crecer interiormente sino en el universo especulativo del lenguaje ante cuya visión nos encontramos y nos reconocemos(...)Pero la palabra no sólo transmite la memoria $y$, por consiguiente, la memoria del pasado sino que, desde ella, se puede organizar el porvenir. Al ser el lenguaje la aportación definidora del ser humano, su cultivo y crítica nutre el principio sobre el que se funda también el desarrollo individual y, en consecuencia, su posible futuro". Y, siendo ello así, para recoger en su lenguaje la memoria y la experiencia personales de Cabrera, y, con él, exaltar su figura en esta conmemoración. Aprovechó Cabrera cuantas oportunidades se le ofrecieron para hacer historia de las etapas más brillantes de la ciencia española del pasado; para participar en la polémica sobre los motivos de nuestro atraso científico; para señalar la importancia del reintegro a nuestro país de los científicos que llevaron a cabo en el extranjero estancias para su especialización; para criticar los obstáculos administrativos a la gestión investigadora; para difundir los momentos más sobresalientes del pensamiento científico en la física y recoger los aspectos de la ciencia de mayor repercusión nacional; para subrayar la distinción entre los menesteres científicos y los políticos; para alabar las actitudes éticas en las publicaciones científicas. Y pocas oportunidades para todo ello como las que tuvo Cabrera en sus discursos de ingreso en la Academias de Ciencias (1910) y la Española (1936), así como en los discursos de contestación a Sánchez Cuervo (1925); del Campo (1927), Palacios (1932) y Moles (1934). 
Hace unos momentos que quedó reseñada la toma de postura de Echegaray en la interpretación del retraso científico español; fue con motivo de su contestación al discurso de ingreso de Cabrera en la Academia de Ciencias. Un cuarto de siglo después, en el discurso de Cabrera, contestación al de ingreso de Moles, insistió en los mismos argumentos: "Muchos de nosotros vinimos a la vida consciente, en una época en que pasaba como evidente la incapacidad del español para la investigación científica; peregrina idea que no dudaron en sostener algunos preclaros hombres que por otros conceptos honran la cultura española. Era un modo fácil de explicar nuestra pobre contribución al progreso científico de Europa en los últimos tres siglos, y, además, una manera cómoda de acallar las acusaciones de nuestra conciencia colectiva por la responsabilidad en que incurrimos al ser meros usuarios de las ventajas de la civilización. No faltaron contradictores a semejante tesis, $y$ aunque inicialmente la fortuna no les acompañó, ha llegado el momento en que nadie osa sostenerlan.

Y en otra ocasión señaló: "Percibo con toda claridad que el interés de este momento radica en hacer propaganda de la Ciencia, contribuyendo a despertar el deseo de su estudio, tanto para la recluta de sus futuros elaboradores, como para la creación de un ambiente que haga posible su vidan. Fueron palabras de Cabrera en la sesión inaugural del curso 192122 en la Real Academia de Ciencias. Eran palabras válidas en aquel segundo intento, para España y para la Física, de convergencia europea, que lideró Cabrera. Son palabras que continúan siendo válidas, para España y para la Ciencia, en nuestro tercer intento iy ojalá sea el último! de incorporación a la ciencia mundial. Son palabras a las que siguieron otras, igualmente servibles en nuestros días: "Gran número de los hombres que consumen su existencia en el laboratorio o gabinete de trabajo, haciendo avanzar lentamente las fronteras de nuestro conocimiento, son soldados de filas que contribuyen con su esfuerzo a la victoria de la Ciencia contra la Naturaleza, en la batalla librada para arrancarle sus secretos. Sea cual fuere la extensión del campo cubierta por la labor de cada uno, su esfuerzo es necesario para el buen éxito; y ciertamente será tanto más entusiasta, cuanto más clara noción tenga del valor de su contribución y más favorable el ambiente que le rodea. En España quizá sea hoy lo más urgente, crear este ambiente para dar mayor impulso al adelantamiento de la ciencia nacional, ya iniciado. Hacer obra útil para conquistar el respeto y la consideración del mundo sabio en medio de una sociedad absolutamente indiferente, sin recibir el calor que da la crítica favorable o adversa de quienes inmediatamente nos rodean, es cosa que 
sólo pueden realizar mentalidades fuera de la medida común, que por desdicha para la Humanidad son bien escasas, lo mismo en España que fuera de nuestra patrian.

Trató Cabrera, en sus manifestaciones públicas, de numerosos argumentos de la política científica de aquellos años, y que, de igual manera, siguen estando vigentes, o quizá aún más, en nuestros mismísimos días. Uno de los mejores documentos para el estudio de la historia de la química fue, y continua siéndolo, el discurso de ingreso en la Academia de Ciencias de don Enrique Moles. Y en la contestación de Cabrera, posiblemente la última de sus intervenciones académicas, aseguró: "Habréis podido apreciar que el trabajo de Moles tiene dos aspectos igualmente interesantes. Es el uno la crítica atenta de la obra científica de los químicos españoles de una época en que estuvimos muy próximos a la incorporación a la vida científica europea. Martí, Elhuyar, Orfila, Carbonell y del Río merecen de parte de Moles la más elevada estima, llegando a calificar la memoria sobre el análisis del aire del primero y la del descubrimiento del wolfram por el segundo, como trabajos dignos de emparejar con las más importantes publicaciones en las ciencias químicas y desde luego superiores a sus contemporáneas. El otro aspecto del discurso de Moles es el análisis que realiza de las condiciones en que desenvolvieron su actividad aquellos hombres beneméritos y las lecciones que su historia puede suministrar a quienes tienen o tengan en el porvenir la responsabilidad aneja a la conducción de la cultura nacional. A la pretendida incapacidad del español para la investigación científica, opone Moles la convicción de que la rémora que ha obstaculizado nuestra colaboración radica en la Administración pública. Fundamenta su creencia con muy interesantes episodios de la vida de aquellos hombres, como la curiosa historia de las obras del que debió ser laboratorio de Proust en Madrid, tan semejante a la de otra construcción en nuestros días, o como la contestación del ministro Ceballos a Orfila, cuando éste hubo de imponer condiciones para venir a regentar la cátedra que le fuera ofrecida en $M a-$ dridn.

Me parece que muy pocos, si alguno, estarán hoy en desacuerdo con este texto de' Cabrera recogiendo la opinión de Moles. Nadie hay que dude de la absoluta y completa capacidad del español para las tareas de todo tipo de creación, ya sea artística, ya sea científica. Nadie habrá tampoco que dude del origen de nuestros obstáculos. Y por si hubiera que aclararlo, Cabrera continúa: "¿Pero qué significa propiamente esto que Moles llama dificultades de la Administración? ¿Hemos de concretar la responsabilidad a los gestores de esa administración, jefes de negociado o 
ministros? Creo yo que son las primeras víctimas porque es difícil no personificar en ellos la responsabilidad de un estado de cosas ciertamente lamentable. Imaginemos por un momento que en el mismo negociado que ha sido la muralla que obstaculizó una acción provechosa para el surgimiento científico de España, sentamos a un hombre comprensivo que allane los obstáculos. Habremos ganado una instancia, pero vendrá otra y después otra. Sólo con un cambio de fondo que supone la renovación de toda la vida oficial se hallaría remedio a este mal innegable. Pero tal renovación no puede ser una simple revolución. Requiere un estado de cultura cuya construcción será la obra lenta de la Universidad».

Permítanme que insista en que cuando Cabrera, presidente de la Academia de Ciencias (1934-38), reflexiona de esta manera es en 1934, el mismo año en que murió Ramón y Cajal, y es el líder e impulsor principal de la investigación en los campos de la física. A pesar de estas críticas, todavía se mantenía la ilusión del acercamiento a la ciencia mundial; ilusión que, en los últimos renglones del mencionado discurso de contestación al ingreso académico de Moles, se manifestaba así: "Si todos ponemos en el esfuerzo el tesón y la actividad de Moles, quizá en nuestros días o en la generación que inmediatamente nos siga, la vida de los investigadores españoles marche por la senda fácil que no pudieron soñar aquellos héroes de los comienzos del siglo pasado, pero sería injusto negar que ya nosotros vislumbramos. Exige un poco de resignación por nuestra parte, pero no veo otro modo de llegar a esa situación que codiciamos. No será para nosotros, pero gozarán de ella nuestros hijos y discípulos».

No hace falta insistir demasiado en cómo se esparcieron estos esfuerzos y estas ilusiones, desaparecieron o se aventaron muchos de los nombres que las mantenían y que, indudablemente, se produjo una discontinuidad en nuestra incorporación al pensamiento científico moderno. No hace falta reiterar tampoco que ha habido que aguardar a una tercera ocasión en la que intentar la convergencia científica con Europa, y que, en mi opinión, nos encontramos actualmente en el torbellino de este nuevo intento, con altibajos y desigualdades, con progresos y con tremendas deficiencias económicas y estructurales; con mejoras absolutas indiscutibles en muchísimos campos, pero con muy discutibles avances relativos a los que ocurren en el resto de los países europeos; con una muy pobre incorporación del cuerpo investigador a la empresa productiva; aunque se haya despenalizado la conexión con la industria, aún con una carencia extraordinaria de relaciones entre la universidad y la investigación y la empresa pública o privada; con muy severas faltas de engarce entre la 
investigación y los investigadores de la ciencia básica y los organismos capaces de su utilización en el sistema global productivo de la nación; con graves carencias en las políticas de personal investigador a lo que añadir incongruencias tremendas en el sistema universitario, incluidos sus planes de estudio. Situación de conjunto, de muchísima mayor envergadura cuantitativa que la que Cabrera entreviera hace seis décadas, pero aún con problemas cualitativos semejantes o agravados; problemas que se recogen en un libro reciente de Primo Yúfera, titulado INTRODUCCION A LA INVESTIGACION CIENTIFICA Y TECNOLOGICA, en párrafos al estilo de los siguientes: "El tercer ciclo universitario malvive y se sostiene gracias a la vocación de algunos profesores. Y esto sucede siendo el tercer ciclo la columna vertebral de la universidad, la fuente de su excelencia y la fuente de energía capaz de elevar el tono cultural, tecnológico, económico y político de la sociedad (...)La universidad española está enferma de un mal grave, el cual tendría un tratamiento quirúrgico, difícil pero efectivo, si no afectara a una parte demasiado extensa de su organismo..." Comentarios que pueden darnos una idea acerca de la discutible, al menos, situación de este tercer intento de convergencia en el que nos encontramos o se encuentran las generaciones siguientes de discípulos de don Blas Cabrera.

Ha cambiado mucho, en efecto, en el último medio siglo, el tema de las publicaciones científicas en general y, en particular, de las publicaciones en español. Fue Cabrera Presidente de la Sociedad Española de Física y Química durante más de una década, y como tal y personalmente contribuyo de forma extraordinaria a la publicación en sus ANALES de la creación científica española. Cabrera mismo, Moles, del Campo, Palacios, Catalán, y los mejores físicos y químicos españoles publicaron preferentemente en los Anales de la Sociedad, lo que no fue obstáculo a que lo mejor de sus aportaciones viera también la luz en otras revistas internacionales. Duro contraste con la situación actual en que, en realidad, se penaliza al científico español en la consideración oficial por publicar en su propia lengua y en sus propias revistas; y hay, sin duda, otras maneras para proteger y estimular, y para evaluar incluso, la calidad investigadora que la simple medida de la lengua extranjera en que aparecen las publicaciones.

Pero, aparte de esta simple aunque importante circunstancia, es asimismo cierta y divulgada la situación, incorrecta muchas veces e incluso inmoral en algunas otras, en que el peso de las publicaciones, y no su calidad y categoría, es la medida administrativa del éxito creador del hom- 


\section{Blas Cabrera, las Academias y su Tiempo}

bre de ciencia. No digamos de las frecuentes ocasiones en que entran en juego los juicios interesados de los censores, las prioridades alteradas por retrasos conscientes, el plagio de resultados, la ocultación sistemática, etc. Se refería Cabrera, a este propósito, al problema teórico que plantearon los multipletes descubiertos por del Campo, cuya interpretación fue dada por Russell y Saunders atribuyendo la superior emisión de energía en algunas líneas espectrales como fruto de la caída simultánea de dos electrones. De esta forma lo comentaba Cabrera, en el discurso de contestación académica a del Campo: "Lo único lamentable es que del Campo no haya recogido los laureles a que pudo tener derecho. Y no es que él no diera ninguna noticia de su labor. Publicó una parte, recogida por Sommerfeld en la cuarta edición de su obra magistral ESTRUCTURA ATOMICA Y LINEAS ESPECTRALES, reservándose otra en espera de un estudio más acabado; pero antes de darle cima, Russell y Saunders dieron a conocer lo que del Campo había callado. Esto, que en otro cualquiera hubiese producido un profundo disgusto, no inmutó a nuestro amigo, en quien pudo más la alegría de ver comprobado su descubrimiento que el natural desconsuelo por la pérdida de la paternidad. Ello prueba un alto temperamento moral ante el cual debemos descubrirnos....."

Por todos lados, y en cualquiera de las múltiples manifestaciones personales, adoptaba Cabrera una gran liberalidad en las opciones de pensamiento. Fue, por ejemplo, en el recuerdo que dedicó al quinto presidente de la Academia de Ciencias, don Amós Salvador y Rodrigañez (1916-22) y a su sucesor en la medalla, don Luis Sánchez Cuervo (192536), con motivo del discurso de contestación a este último en su toma de posesión académica. De este discurso son los siguientes párrafos: "Antes que esta posesión de la medalla número 19, y que el título de ingeniero de Caminos, existe entre estos dos hombres una comunidad de afanes por el engrandecimiento de la Patria, aunque los medios que uno y otro han utilizado sean radicalmente opuestos, que no en vano pertenecen los Srs. Salvador y Sánchez Cuervo a generaciones distantes casi medio siglo en el tiempo, y mucho más en los ideales que las han animado. Vino a la vida el Sr. Salvador en el periodo de nuestra historia en que el talento consideraba como su ocupación más excelsa dar realidad a unos ideales politicos, por los cuales los hombres de la época se jugaban frecuentemente la vida. El Sr. Sánchez Cuervo pertenece, por el contrario, a una generación que se aparta voluntariamente de los menesteres de la politica y aplica sus actividades en el campo de la Ciencia, o de la Industria, o de las Artes, o de los otros múltiples aspectos de la vida, que es mucho más compleja de lo que pensaban nuestros abuelos y nuestros padres. Creían ellos 
servir más eficazmente a la Patria organizando su Constitución política, ya de acuerdo, ya en oposición con los ideales proclamados por la Revolución francesa. Por el contrario, estima nuestra generación que lo fundamental en los pueblos no es la forma externa, que representa su Constitución política, sino la riqueza de su contenido en aquello que es característico de la vida moderna: la abundancia de su aportación al progreso humano en todos sus órdenes!r.

Se apuntaba, sin duda, Cabrera a los gustos de Sánchez Cuervo cuando, a continuación, afirmaba: "Asombraría a nuestros abuelos el que un hombre de la altura mental del Sr. Sánchez Cuervo no se sienta tentado por la política y prefiera la confección de un proyecto de electrificación de ferrocarriles a modificar, en un sentido más liberal o conservador, nuestro Código fundamental. En cambio, miramos muchos de nosotros con pena las energías malgastadas por muchas inteligencias de primer orden del pasado siglo en la discusión de problemas que se nos antojan completamente secundarios. Y no es que sintamos menos que ellos las libertades individuales o la independencia de la Patria; es que sabemos que las unas y la otra no se conquistan y conservan sino con el fomento de la cultura $y$ el mejoramiento material. Son inútiles las libertades cuando no se saben usar, y es ilusoria la independencia mientras seamos tributarios del extranjero en las más elementales exigencias de la vida contemporánea. Por esto yo aplaudo con entusiasmo a nuestro compañero cuando le veo trabajar con ahínco por la utilización más completa de los factores de riqueza que la Naturaleza nos brinda, no sólo formulando proyectos inmediatamente realizables, sino haciendo obra de propaganda en las publicaciones profesionales de nuestro país en pro de una transformación en sentido moderno de nuestra industria nacional».

A través de estos cuantos retazos de la actitud científica y humana de Cabrera, se puede gustar mejor, sin duda, de su personalidad que por medio de los temas científicos particulares que experimentalmente cultivó. Tuvo Cabrera, sin embargo, especial apego a los planteamientos generales de la EVOLUCION DE LOS CONCEPTOS DE LA FISICA; y a ello se debió, en 1921, el Discurso de Inauguración del Curso académico en la Real de Ciencias, así como el Discurso de ingreso en la Española, ya en 1936. Fueron ambos, modelos del magisterio que permanentemente ejerció sobre los jóvenes físicos durante un brillante cuarto de siglo de la ciencia española. Y sobre la ciencia y la cultura españolas desde sus mejores cumbres, la Academia y la Universidad, la Sociedad de Física y Química y el Laboratorio de Investigaciones físicas de la Junta, las numerosas publicaciones especializadas y su destacado reconoci- 


\section{Blas Cabrera, las Academias y su Tiempo}

miento internacional, en un clima nada propicio que él contribuyó como pocos a transformar.

Si ya hemos mencionado cómo las ilusiones, los esfuerzos e, incluso, sus artífices se vieron resquebrajados y dispersos, de forma que aquel simple embrión no pudo ni siquiera anidar en una fisiológica matriz social; Cabrera contribuyó personalmente a contrarrestar sus indudables consecuencias, y a mantener una imagen exterior, posiblemente muy débil y con toda seguridad deformada, pero sobre la que, con muchísimo esfuerzo, se ha ido forjando durante muchas décadas la realidad presente. A ello colaboró de manera extraordinaria Cabrera desde la dirección de la Universidad de Verano de Santander. En 1933 acudieron a ella Euler, Haber y Willstätter; y al año siguiente lo hizo Schrödinger, con quien Cabrera inició una relación personal de la que fue fruto una interesante correspondencia. Y, precisamente, el 28 de marzo de 1937, en una carta escrita en castellano, se preocupaba Schrödinger de sus amigos españoles de esta manera: "...Excuso decir que estábamos muy inquietos por Vds. todos y muy afligidos por lo que se lee en los periódicos por todo este tiempo sobre la inefable desgracia de nuestra querida España. No nos atrevimos escribirles ya que en una época alborotosa como ésa nunca se puede saber qué efecto, tal vez desgraciado o siquiera molesto, vaya a salir de una carta llegando del extranjero (...) ¿Y qué tal, cómo lo pasan Vds.? ¿Qué se hará de su magnifico Instituto? ¿Hay alguna esperanza de volver Vd. allá? Me figuro que son estas las mismas preguntas que Vd. se pone diariamente sin poder contestarlas". 